University of Nebraska - Lincoln

DigitalCommons@University of Nebraska - Lincoln

2008

\title{
Association and Structure of Thermosensitive Comblike Block Copolymers in Aqueous Solutions
}

\author{
Gang Cheng \\ Fengjun Hua \\ Yuri B. Melnichenko \\ Kunlun Hong \\ Jimmy W. Mays \\ See next page for additional authors
}

Follow this and additional works at: https://digitalcommons.unl.edu/usdoepub

Part of the Bioresource and Agricultural Engineering Commons

Cheng, Gang; Hua, Fengjun; Melnichenko, Yuri B.; Hong, Kunlun; Mays, Jimmy W.; Hammouda, Boualem; and Wignall, George D., "Association and Structure of Thermosensitive Comblike Block Copolymers in Aqueous Solutions" (2008). US Department of Energy Publications. 77.

https://digitalcommons.unl.edu/usdoepub/77

This Article is brought to you for free and open access by the U.S. Department of Energy at DigitalCommons@University of Nebraska - Lincoln. It has been accepted for inclusion in US Department of Energy Publications by an authorized administrator of DigitalCommons@University of Nebraska - Lincoln. 


\section{Authors}

Gang Cheng, Fengjun Hua, Yuri B. Melnichenko, Kunlun Hong, Jimmy W. Mays, Boualem Hammouda, and George D. Wignall 


\title{
Association and Structure of Thermosensitive Comblike Block Copolymers in Aqueous Solutions
}

\author{
Gang Cheng, ${ }^{*}{ }^{\dagger}$ Fengjun Hua, ${ }^{\ddagger}$ Yuri B. Melnichenko, ${ }^{\dagger}$ Kunlun Hong, ${ }^{\ddagger}$ Jimmy W. Mays, ${ }^{\ddagger}$ \\ Boualem Hammouda, ${ }^{\S}$ and George D. Wignall ${ }^{\dagger}$
}

Neutron Scattering Science Division and Center for Nanophase Materials Sciences, Oak Ridge National Laboratory, Oak Ridge, Tennessee 37831, and National Institute of Standards and Technology, 100 Bureau Drive, Gaithersburg, Maryland 20899-6102

Received November 20, 2007; Revised Manuscript Received April 16, 2008

\begin{abstract}
The structures and association properties of thermosensitive block copolymers of poly(methoxyoligo(ethylene glycol) norbornenyl esters) in $\mathrm{D}_{2} \mathrm{O}$ were investigated by small angle neutron scattering (SANS). Each block is a comblike polymer with a polynorbornene (PNB) backbone and oligo ethylene glycol (OEG) side chains (one side chain per NB repeat unit). The chemical formula of the block copolymer is $\left(\mathrm{OEG}_{3} \mathrm{NB}_{79}-\right.$ $\left(\mathrm{OEG}_{6.6} \mathrm{NB}\right)_{67}$, where subscripts represent the degree of polymerization (DP) of OEG and NB in each block. The polymer concentration was fixed at $2.0 \mathrm{wt} \%$ and the structural changes were investigated over a temperature range between 25 and $68{ }^{\circ} \mathrm{C}$. It was found that at room temperature polymers associate to form micelles with a spherical core formed by the block $\left(\mathrm{OEG}_{3} \mathrm{NB}\right)_{79}$ and corona formed by the block $\left(\mathrm{OEG}_{6.6} \mathrm{NB}\right)_{67}$ and that the shape of the polymer in the corona could be described by the form factor of rigid cylinders. At elevated temperatures, the aggregation number increased and the micelles became more compact. At temperatures around the cloud point temperature (CPT) $T=60^{\circ} \mathrm{C}$ a correlation peak started to appear and became pronounced at 68 ${ }^{\circ} \mathrm{C}$ due to the formation of a partially ordered structure with a correlation length $\sim 349 \AA$.
\end{abstract}

\section{Introduction}

The ability of amphiphilic block copolymers to form micelles with tunable dimensions and structures has triggered extensive research activities over the past few decades. ${ }^{1,2}$ The structure of block copolymer micelles may be manipulated by varying different parameters such as the molecular weight of each block, the overall molecular weight, polymer concentration, temperature, solvent selectivity, etc. Structural characterization techniques such as small angle neutron scattering (SANS), light scattering, and transmission electron microscopy have shown that block copolymers associate in solutions with a variety of structures (spheres, cylinders, bilayers, etc.) with insoluble blocks forming a "core" separated from the solvent by an outer shell or "corona" formed by soluble blocks. ${ }^{3-6}$ Recently, it has been demonstrated that the morphology of micelles can be changed by using mixed solvents without changing the chemistry of the block copolymers. ${ }^{4-6}$ Similarly, studies ${ }^{2,7}$ revealed novel structures in solutions of nonlinear block copolymers. Thus, altering the architecture of constituting blocks may provide an additional important tool for tuning the resulting micellar morphology and expand number of options available for nanoscale technologies.

Linear polymers grafted with OEG dendrons have been shown to associate to form nanofibers ${ }^{8}$ in water. These polymers are thermosensitive and potentially biocompatible due to the presence of EG segments, which makes them of interest for various applications such as drug delivery, "smart" surfaces, etc. ${ }^{9,10} \mathrm{New}$ thermosensitive polymers with two or more thermo sensitive blocks and controllable cloud point temperatures (CPTs) have been recently synthesized and studied. ${ }^{11-13}$ The existence of CPTs at which individual polymers or micellar solutions experience abrupt structural changes was demonstrated. If each

\footnotetext{
* Corresponding author.

Neutron Scattering Science Division, Oak Ridge National Laboratory.

Center for Nanophase Materials Sciences, Oak Ridge National Laboratory.

$\S$ National Institute of Standards and Technology.
}

of the blocks has a different CPT, the block copolymers are expected to self-assemble in solution and form micelles at temperature close to the CPT of one of the blocks. ${ }^{11,13}$ Thus, aqueous solutions of poly(methoxytri(ethylene glycol)acrylate)poly(4-vinylbezyl methoxytris(oxyethylene ether) block copolymers, revealed the existence of two CPTs, induced by dehydration of one of the blocks and the collapse of the other block. ${ }^{13}$ In this paper we report results of the SANS study of the structure and association of the OEG-grafted PNB block copolymers in aqueous solutions at different temperatures. At low temperatures $\left(25{ }^{\circ} \mathrm{C}<T<52{ }^{\circ} \mathrm{C}\right.$ ) the polymers form micelles with a spherical core and a shell with cylinder-like water soluble polymers. Above the $\mathrm{CPT}\left(60^{\circ} \mathrm{C}\right)$ the micelles associate to form partially ordered structures with a correlation length of $\sim 349$ A.

\section{Experimental Section}

Materials. The chemical structure of the block copolymer studied is shown in Figure 1, and the chemical formula of the block copolymer is $\left(\mathrm{OEG}_{3} \mathrm{NB}\right)_{79}-\left(\mathrm{OEG}_{6.6} \mathrm{NB}\right)_{67}$, where subscripts denote the DP of OEG and NB in each block with the polydispersity of 1.26 , as determined by gel permeation chromatography. The scattering length densities (SLD) were calculated under the assumption that the density of the backbone is that of the bulk PNB $\left(1.35 \mathrm{~g} / \mathrm{cm}^{3}\right)$ and the density of the side chains is that of the bulk

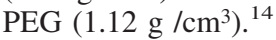

SANS Measurements. SANS measurements were performed at the National Institute for Standards and Technology Center for Neutron Research on the NG7 30 m instrument with a neutron wavelength of $\lambda=6.0 \AA$ ( $\Delta \lambda / \lambda \sim 0.15)$. Two sample-detector distances were used (1.5 and $13.0 \mathrm{~m}$ with a $25 \mathrm{~cm}$ detector offset), which resulted in the overall range of the scattering vector $(q)$ covered in the experiments $0.0042 \AA^{-1}<q=4 \pi \lambda^{-1} \sin \theta<0.40$ $\AA^{-1}$, where $2 \theta$ is the scattering angle. The data were corrected for instrumental background as well as detector efficiency and put on absolute scale [cross section $I(q)$ per unit volume in units of $\mathrm{cm}^{-1}$ ] based on measurements of the flux of the direct beam. The scattering from the solvent was measured and subtracted according to its volume fraction. The scattering function used to fit the SANS data in this study is given in the Appendix. The calculated SLD of 


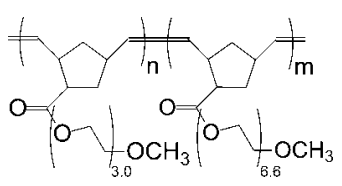

Figure 1. Chemical structure of block copolymer $\left(\mathrm{EG}_{3} \mathrm{NB}\right)_{79}-$ $\left(\mathrm{EG}_{6.6} \mathrm{NB}\right)_{67}$.

the $\left(\mathrm{EG}_{3} \mathrm{NB}\right)_{79}$ block is $\sim 7.9 \times 10^{-7} \AA^{-2}$ and that of the $\left(\mathrm{EG}_{6.6} \mathrm{NB}\right)_{67}$ block is $\sim 8.4 \times 10^{-7} \AA^{-1}$, and therefore, there is virtually no contrast between the two blocks. The temperature range covered in the experiments was varied between 25 and $68{ }^{\circ} \mathrm{C}$ and was controlled to better than $\pm 0.2{ }^{\circ} \mathrm{C}$ using a water circulation bath. Before starting the measurements, solutions were equilibrated at each temperature for at least $60 \mathrm{~min}$. The run times at each temperature at 13.0 and $1.5 \mathrm{~m}$ sample-detector distances were 15 and $5 \mathrm{~min}$, respectively.

\section{Results and Discussion}

At a fixed length of the hydrophobic PNB backbone, the solubility of the OEG-PNB polymer in water can be tuned by varying the number of hydrophilic EG repeat units in the side chains. When the number of EG repeat units is low (e.g., 3), the OEG grafted PNB homopolymer is found to be nonsoluble in water even down to the lowest temperatures $\left(\sim 4{ }^{\circ} \mathrm{C}\right)$ just above the freezing point. Thus, the block with $\left(\mathrm{OEG}_{3} \mathrm{NB}\right)_{79}$ is essentially hydrophobic at all temperatures. If the DP of OEG side chains increases above certain threshold value $(\sim 4)$ the homopolymer becomes water soluble and thus the block with the DP of OEG $6.6\left(\mathrm{OEG}_{6.6} \mathrm{NB}\right)_{67}$ is hydrophilic. Due to the different solubility of the two blocks in water, one might expect $\left(\mathrm{OEG}_{3} \mathrm{NB}\right)_{79}-\left(\mathrm{OEG}_{6.6} \mathrm{NB}\right)_{67}$ block copolymers associate and form micelles with a core of hydrophobic $\left(\mathrm{OEG}_{3} \mathrm{NB}\right)_{79}$ blocks and a shell of the hydrophilic $\left(\mathrm{OEG}_{6.6} \mathrm{NB}\right)_{67}$ polymers. Only recently micelles formed by amphiphilic block copolymer polystyrene-grafted polystyrene (PS- $g$-PS) and polyisoprenegrafted polyisoprene (PI- $g$-PI) with hydrophobic PS- $g$-PS in the core and hydrophilic PI- $g$-PI in the shell were observed for the first time ${ }^{7}$ however, the conformation of both blocks in the micelles was not investigated. In our recent paper, we studied the conformation of an $\left(\mathrm{EG}_{6.6} \mathrm{NB}\right)_{50}$ homopolymer with chemical composition and DP of the backbone and side chains similar to those of one of the blocks of the block copolymer studied in this work. It was shown that $\left(\mathrm{EG}_{6.6} \mathrm{NB}\right)_{50}$ homopolymers assume the shape of rigid cylinders when dissolved in $\mathrm{D}_{2} \mathrm{O} .{ }^{14}$ On the basis of this experimental observation, it is reasonable to expect that the shape of the water soluble block $\left(\mathrm{EG}_{6.6} \mathrm{NB}\right)_{67}$ in the outer shell of the micelles may also be approximated by the form factor of rigid cylinders.

SANS data from the $2.0 \mathrm{wt}$ \% block copolymer solution in $\mathrm{D}_{2} \mathrm{O}$ taken at several temperatures are shown in Figure 2. The upturn in the low $q$ range of 0.003 to $0.005 \AA^{-1}$ indicates the presence of large clusters. The scattering in the intermediate $\mathrm{q}$ range of $0.005 \AA^{-1}<q<0.03 \AA^{-1}$, corresponds to the scattering from individual micelles whereas in the high $q$ range of 0.03 $\AA^{-1}<q<0.2 \AA^{-1}$ it mainly comes from the local concentration fluctuations inside the micelles. What is different from the widely studied linear block copolymer micelles is that the scattering intensity follows a much steeper decay than -2 starting from $q=0.13 \AA^{-1}$, which is believed to be related to the cylindrical structures in the shell. With increase of temperature, the scattering intensity in the intermediate $q$ regime increases, which is attributed to the increase of aggregation number of the micelles. The small decrease of scattering intensity in the high $q$ regime from 25 to $52^{\circ} \mathrm{C}$ suggests that the local conformation of the block copolymers in the micelles changes only slightly. Previous studies of poly(ethylene oxide)poly(propylene oxide)-poly(ethylene oxide) (PEO-PPO-PEO)

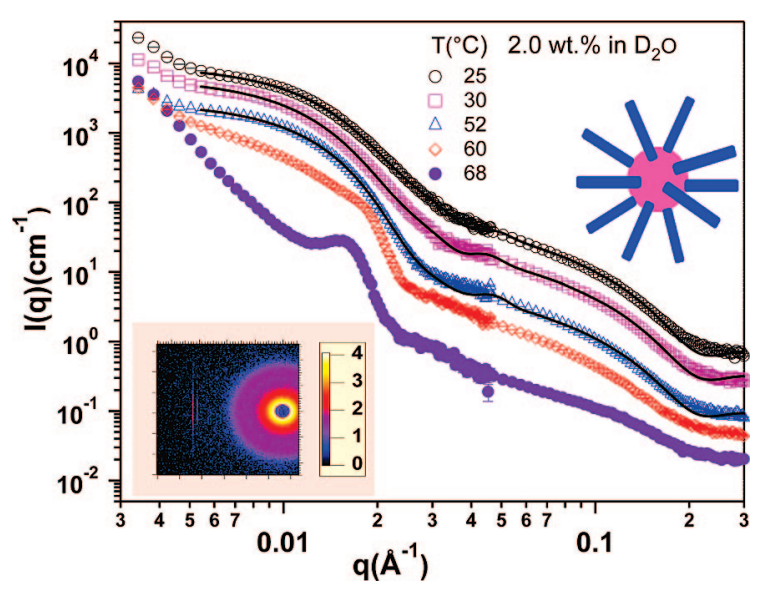

Figure 2. SANS data from $2.0 \mathrm{wt} \%\left(\mathrm{EG}_{3} \mathrm{NB}\right)_{79}-\left(\mathrm{EG}_{6.6} \mathrm{NB}\right)_{67}$ block copolymer in $\mathrm{D}_{2} \mathrm{O}$ as a function of temperature. The error bars are smaller than the size of the symbols. The data of $25,30,52$, and $60{ }^{\circ} \mathrm{C}$ are shifted up by factos of $30,14,4$, and 2 respectively for the sake of clarity. The lower left inset is a two-dimensional detector image of the scattering from the studied polymer solution at $68^{\circ} \mathrm{C}$. The upper right inset is a schematic illustration of the proposed structure of the micelles.

triblock copolymer micelles in water also demonstrated a small variation ${ }^{15,16}$ or no variation ${ }^{17}$ in scattering intensity in the high $q$ regime. At temperatures close to the solution CPT $\left(60^{\circ} \mathrm{C}\right)$, the scattering pattern changes dramatically in the intermediate $q$ range and a broad peak starts to develop. The micelles inside the clusters begin to exhibit a certain degree of spacial ordering, which eventually manifests itself as appearance of the "correlation peak" at $68{ }^{\circ} \mathrm{C}$ with the maximum corresponding to $0.018 \AA^{-1}$ and the correlation length $2 \pi / 0.018\left(\AA^{-1}\right)=349 \AA$. In the dilute solutions, block copolymer micelles are often in a disordered state. However they may self-organize to form "lattices" when the concentration is high enough. ${ }^{18}$ SANS from the dilute turbid aqueous solutions of micelles of PEO-PPOPEO triblock copolymers at temperatures around the CPT revealed a broad peak in the SANS curves. ${ }^{15,19}$ Large, micrometer-sized clusters were detected in the solution and micelles inside the clusters were organized to form a partially ordered structure, ${ }^{19}$ and this observation is in agreement with the results reported in our study. As may be seen in the 2D scattering pattern shown in the inset in Figure 2, the solution remains isotropic at $68^{\circ} \mathrm{C}$ and no significant amount of polymers precipitates to the bottom at this temperature, as confirmed by independent measurements of the neutron transmission.

Guinier analysis of the SANS data in the $q$ range of 0.005 $\AA^{-1}<q<0.01 \AA^{-1}$ gives the scattering cross section at zero angle, $I(0)$, and the radius of gyration of individual micelles, $R_{\mathrm{g}}$, which are shown in Figure 3, parts a and b, respectively. The errors generated from the fitting are small; and therefore a $10 \%$ error was added to the obtained value of $I(0)$ considering the uncertainties of the absolute scaling. The errors associated to the $R_{\mathrm{g}}$ of the micelles, $7-8 \%$, were estimated based on the obtained polydispersity of the core radius through modeling of the SANS data, which is descried later. The $I(0)$ is proportional to the aggregation number of a micelle and its value increases with temperature. The average values of $R_{\mathrm{g}}$ drops by about $10 \%$ over the temperature range of 25 to $52{ }^{\circ} \mathrm{C}$, indicating that the micelles are contracting slightly with temperature.

The micellar structure may be assessed more quantitatively by fitting the SANS data to an appropriate model. Previous SANS studies of water soluble block copolymers were mainly focused on triblock copolymer PEO-PPO-PEO in water. ${ }^{15-20}$ SANS data in previous studies were fitted to several models, which fall into three general categories: a homogeneous sphere, 

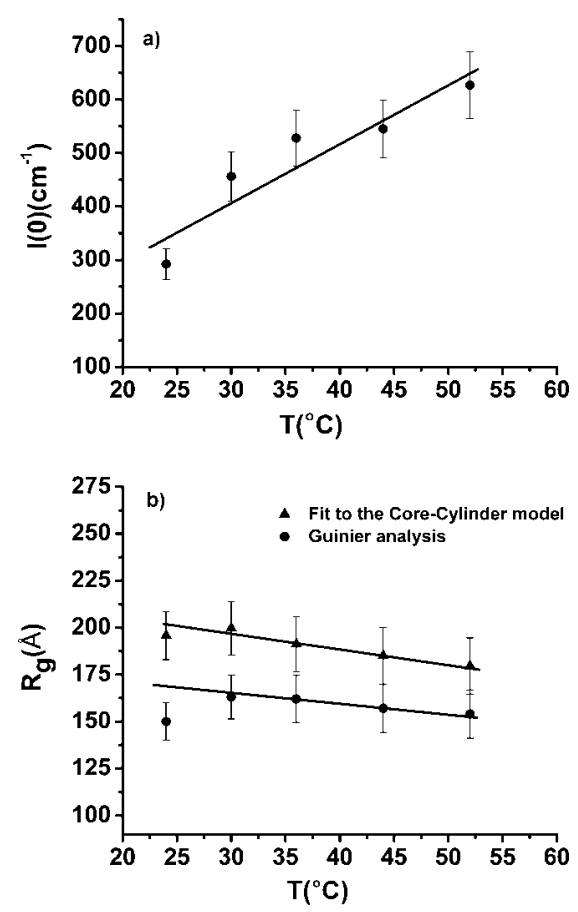

Figure 3. Results of the Guinier analysis of the SANS data of the block copolymers in $2.0 \mathrm{wt} . \% \mathrm{D}_{2} \mathrm{O}$. Key: (a) $I(0)$ as a function of temperature; (b) $R_{\mathrm{g}}$ as a function of temperature. The radius of the core of the micelles has a polydispersity of 0.15 based on the modeling of the SANS data, which defined the error bars of the $R_{\mathrm{g}}$ shown in the figure. The solid lines in Figure 3 are guides to the eye.

a homogeneous core + homogeneous shell and a homogeneous core + inhomogeneous shell. The last model was found to represent most closely the real micellar structure. Before fitting, assumptions had to be made regarding the core composition, i.e., whether the core represents pure PPO, PPO with certain amount of water, or PPO with a fraction of PEO. The second assumption was found to be the most plausible as the fitting results correlated with general expectations based on the fundamental physics of micellization such as growth of the aggregation number and decrease of the water concentration in the core with temperature.

The form factor of the block copolymer micelles studied in this work (see the Appendix) is a special case of a previously developed model. ${ }^{21}$ We have modified the formalism given in ref 21 to describe the structure of micelles with a corona consisting of noninteracting chains with form factor of rigid cylinders (see the Appendix), namely core-cylinder model. The fitting parameters are the size of the core, $R_{\text {core }}$, the polydispersity of the core, $\sigma$, the size of the shell (length of the cylinder), $R_{\text {shell }}$, the cross section radius of the cylinder, $R$, and the aggregation number, $N_{\text {agg. }}$. A Schulz distribution ${ }^{22}$ was used to account for the core polydispersity. We assume the core consists of $\left(\mathrm{OEG}_{3} \mathrm{NB}\right)_{79}$ blocks and some amount of water while the $\left(\mathrm{EG}_{6.6} \mathrm{NB}\right)_{67}$ blocks reside in the shell. The resulting fits are shown as solid line in Figure 2 and the values of the fitting parameters are given in Table 1. The fits to the micellar form factor (without intermicellar interactions) gave values of the reduced $\chi^{2}$ in the range $8-10$. Such vauels are common to the modeling of scattering functions of the block copolymer micelles (see, e.g., ref 17) and the major contribution to $\chi^{2}$ is coming from the high $q$ domain. On the other hand, inclusion to the fitting procedure a structure factor should affect the fit mainly in the low $q$ domain, where the quality of the fits is rather satisfactory. This brings us to a conclusion that intermicellar interactions are negligibly small and may be omitted in the model.
Table 1. Fitting Parameters of the Core-Cylinder Model $^{a}$

\begin{tabular}{ccccccc}
\hline$T\left({ }^{\circ} \mathrm{C}\right)$ & $R_{\text {core }}(\AA)$ & $R_{\text {shell }}(\AA)$ & $N_{\text {agg }}$ & $\phi_{\mathrm{p}}$ & $\mathrm{R}(\AA)$ & $s(\AA)$ \\
\hline 25 & 111 & 142 & 36 & 0.21 & 17 & 10 \\
30 & 123 & 135 & 65 & 0.28 & 16 & 13 \\
36 & 128 & 119 & 78 & 0.30 & 16 & 11 \\
44 & 130 & 109 & 96 & 0.35 & 15 & 11 \\
52 & 129 & 103 & 125 & 0.47 & 16 & 14
\end{tabular}

${ }^{a}$ The errors generated from the fitting are small (less than 1\%). The absolute scale of the experiment has about $10 \%$ errors and therefore the aggregation number has about the same error. The radius of the core has a polydispersity of 0.15 . The volume fraction of the polymers in the core is calculated through $\phi_{\mathrm{p}}=N_{\mathrm{agg}} V_{\text {core-polymer }} / V_{\text {core }}$, where $V_{\text {core }}=4 \pi R_{\text {core }}{ }^{3 / 3}$. Taking into account the $10 \%$ uncertainty in the aggregation number radius, the errors associated with $\phi_{\mathrm{p}}$ is about $10 \%$ of its average value.
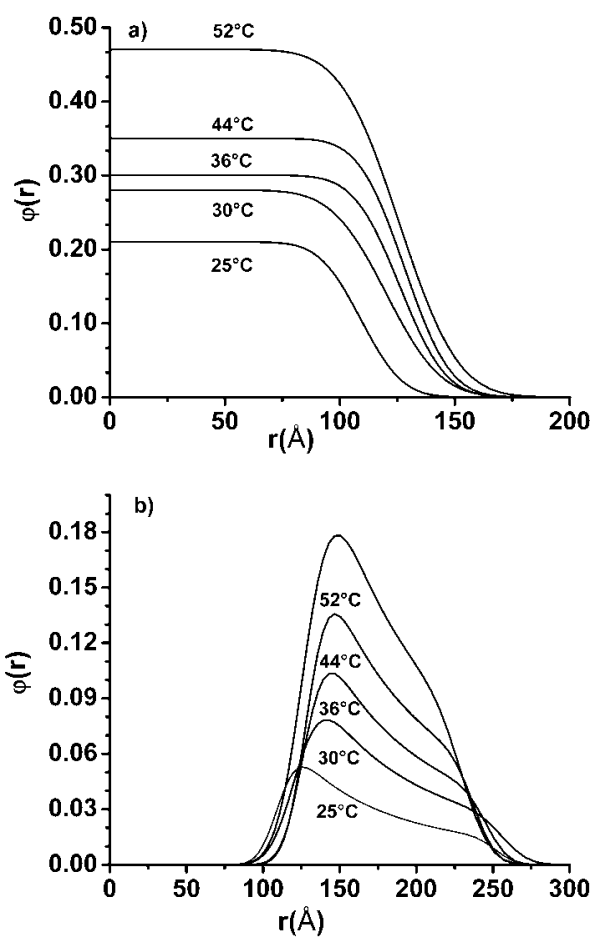

Figure 4. Radial profiles of the polymer volume fraction (a) in the core and (b) in the shell.

As may be seen in Table 1, the aggregation number and the calculated volume fraction of polymers in the core increase with temperature, which is similar to the behavior of PEO-PPO-PEO micelles. The radial profile of the polymers in the core and in the shell is obtained by a Fourier transform of the amplitude of the core and the shell respectively. The calculated profiles are shown in Figure 4 and the equations are given in the Appendix. The average sizes of the micelles are 15-20\% larger than that obtained from the Guinier analysis (Figure $3 b$ ). We note that the values of volume fraction of the polymer in the core is rather low, especially at low temperatures. We believe this to be related to the specifics of the structure of comblike polymers: the availability of the rigid OEG side chains imposes steric restrictions which make it difficult for the $\left(\mathrm{OEG}_{3} \mathrm{NB}\right)_{79}$ block to form a compact core. The core size initially increases and then stays roughly constant while the size of the shell decreases continuously with temperature in the range between 25 and 52 ${ }^{\circ} \mathrm{C}$. Assuming that the length of a norbornyl repeat unit is close to $5 \AA,{ }^{14}$ the contour length of the studied fully extended PNB backbone is $67 \times 5 \AA=335 \AA$. Thus, the $\left(\mathrm{EG}_{6.6} \mathrm{NB}\right)_{67}$ blocks appear to be partially collapsed in the shell. The observed variation of the shell size correlates with the variation of the $\mathrm{R}_{\mathrm{g}}$ obtained from Guinier analysis (Figure $3 \mathrm{~b}$ ) and suggests that the reduction of the micelle dimensions is caused by the contraction of the polymer in the shell due to deteriorating 
solvent quality. The polymers in the shell contract by $\sim 25 \%$ from 25 to $52{ }^{\circ} \mathrm{C}$ which should lead to $\sim 16 \%$ increase of the cross section radius of the cylinders given a fixed volume of each cylinder. It is however found that the cross section radius is not sensitive to the change of temperature and stays approximately constant at $16 \pm 1 \AA$. We explain this result as due to the relatively small value of the cross section radius as well as possible contraction of OEG arms with temperature.

\section{Summary}

Diblock copolymers $\left(\mathrm{EG}_{3} \mathrm{NB}\right)_{79}-\left(\mathrm{EG}_{6.6} \mathrm{NB}\right)_{67}$ associate to form micelles in $\mathrm{D}_{2} \mathrm{O}$ at room temperature. SANS data can be adequately fitted to the model based on a spherical core and rigid cylinders in the corona. Due to the comblike structure, the micellar core of the studied micelles is not as compact as the core of micelles formed by linear polymers. The aggregation number increases and the overall size of the micelles decreases with temperature due to contraction of the polymers in the corona as a result of deteriorating solvent quality. Above the cloud point temperature $\left(T=60^{\circ} \mathrm{C}\right)$, the micelles reveal partial ordering with the correlation length $\sim 349 \AA$.

Acknowledgment. G.C. thanks Professor J. S. Pedesen for the helpful discussions regarding the construction of the scattering functions. Research sponsored by the Division of Materials Sciences and Engineering, Office of Basic Energy Sciences, U.S. Department of Energy, under Contract DE-AC05-00OR22725 with Oak Ridge National Laboratory, managed and operated by UT-Battelle, LLC. Part of this research was conducted at the Center for Nanophase Materials Sciences, which is sponsored at ORNL by the Division of Scientific User Facilities, U.S. Department of Energy. G.C. and F.H. are supported in part by an appointment to the ORNL Postdoctoral Research Associates Program, administered jointly by the ORNL and the Oak Ridge Associated Universities. The identification of any commercial product or trade name does not imply endorsement or recommendation by the National Institute of Standards and Technology, nor does it imply that the items identified are necessarily the best available for the purpose. This work is based upon activities supported in part by the National Science Foundation under Agreement No. DMR-0454672 to the NIST Center for Neutron Research.

\section{Appendix}

The scattering function of a micelle consisting of a spherical uniform core with many noninteracting rigid cylinders attached on its surface is approximated based on the formalism in ref 21. It consists of four terms, the scattering of the spherical core, of the cylinders in the shell, of the interference between the cylinders in the shell and of the interference between the core and the shell. The fitting parameters are the radius of the core, $R_{\text {core, }}$ the size of the shell (length of the cylinder), $R_{\text {shell }}$, the cross section radius of the cylinder, $R$, the aggregation number, $N_{\text {agg }}$.

- The core term, consisting of the form factor of an uniform sphere: $\left[V_{\text {core }}\left(\rho_{\text {core }}-\rho_{\text {solvent }}\right) \Phi(q)\right]^{2}$, where $\Phi(q R)=3[\sin (q R)$ - $q R \cos (q R)] /(q R)^{3}, \rho_{\text {core }}$ is the scattering length density of the core-forming block, and $\rho_{\text {solvent }}$ is the scattering length density of the solvent.

- Scattering due to rigid cylinders in the shell: $N_{\text {agg }}\left[V_{\text {chain }}\left(\rho_{\text {chain }}\right.\right.$ $\left.\left.-\rho_{\text {solvent }}\right)\right]^{2} F_{\text {cyl }}(q)$, where $F_{\text {cyl }}$ is the form factor of a rigid cylinder and $F_{\text {cyl }}=\int_{0}^{\pi / 2}\left[\sin ^{2}(q H \cos \theta) / q^{2} H^{2} \cos ^{2} \theta\right]\left[4 J_{1}^{2}(q R\right.$ $\left.\sin \theta) / q^{2} R^{2} \sin ^{2} \theta\right] \sin \theta \mathrm{d} \theta, H$ is the half-length of the cylinder, $\theta$ is the angle between the vector $q$ and the long axis of the cylinder, $J_{1}(x)$ is the first order Bessel function of the first kind, $\rho_{\text {chain }}$ is the scattering length density of the shell-forming block.

- Scattering due to interferences of the cylinders in the shell: $N_{\text {agg }}\left(N_{\text {agg }}-1\right)\left[V_{\text {chain }}\left(\rho_{\text {chain }}-\rho_{\text {solvent }}\right) A_{\text {chain }}\right]^{2}$, where $A_{\text {chain }}$ is the normalized Fourier transform of the density profile of the shell and $A_{\text {chain }}(q)=\left\{\int_{R_{\text {corer }}}^{R_{\text {micll }} \mathrm{d} r} 4 \pi r^{2} \phi(r)[\sin (q r) /(q r)]\right\} /\left[\int_{R_{\text {core }}}^{R_{\text {miclle }}} \mathrm{d} r 4 \pi r^{2} \phi(r)\right]$, $\phi(r)=1 /\left(4 \pi r^{2}\right)$, is the radial volume fraction profile of a rod in the shell.

- Cross term due to core and shell: $2 N_{\mathrm{agg}}^{2} V_{\text {core }}\left(\rho_{\text {core }}-\right.$ $\left.\rho_{\text {solvent }}\right) V_{\text {chain }}\left(\rho_{\text {chain }}-\rho_{\text {solvent }}\right) A_{\text {chain }}(q) \Phi(q)$.

The form factor is given as $F_{\text {micelle }}(q)=N_{\text {agg }}^{2} \beta_{\text {core }}^{2} \Phi$ $(q)^{2}+N_{\text {agg }} \beta_{\text {chain }}{ }^{2} F_{\text {cyl }}(q)+N_{\text {agg }}\left(N_{\text {agg }}-1\right) \beta_{\text {chain }}{ }^{2} A_{\text {chain }}{ }^{2}+$ $2 N_{\text {agg }}{ }^{2} \beta_{\text {core }} \beta_{\text {chain }} A_{\text {chain }}(q) \Phi(q)$, where $V_{\text {chain }}\left(\rho_{\text {chain }}-\rho_{\text {solvent }}\right)=\beta_{\text {chain }}$, $V_{\text {chain }}=44750 \AA^{3}$ is the volume of the shell- forming block. $V_{\text {core-polymer }}\left(\rho_{\text {core }}-\rho_{\text {solvent }}\right)=\beta_{\text {core }}, V_{\text {core-polymer }}=33881 \AA^{3}$ is the volume of the core-forming block.

Considering the smooth decay of the scattering length density at the core-shell interface, both the form factor amplitude of the core, $\Phi(q)$, and of the shell, $A_{\text {chain, }}$ are multiplied by $\exp \left(-q^{2} s^{2} / 2\right)$, where $s$ corresponds to the width over which the density decays. ${ }^{17}$

The radial volume fraction profile of the polymers in the core is calculated ${ }^{20 \mathrm{~d}}$ by $\phi(r)=\left(1 / 2 \pi^{2}\right) \int_{0}^{\infty} N_{\mathrm{agg}} V_{\text {core-polymer }} \Phi(q)[\sin (q r) /$ $q r] q^{2} \exp \left(-q^{2} s^{2} / 2\right) \mathrm{d} q$ and in the shell is calculated by $\phi(r)=$ $1 / 2 \pi^{2} \int_{0}^{\infty} N_{\text {agg }} V_{\text {chain }} A_{\text {chain }}(q)[\sin (q r) / q r] q^{2} \exp \left(-q^{2} s^{2} / 2\right) \mathrm{d} q$.

\section{References and Notes}

(1) Rodríguez-Hernández, J.; Chécot, F.; Gnanou, Y.; Lecommandoux, S. Prog. Polym. Sci. 2005, 30, 691-724.

(2) Hadjichristidis, N.; Iatrou, H.; Pitsikalis, M.; Pispas, S.; Avgeropoulos, A. Prog. Polym. Sci. 2005, 30, 725-782.

(3) Zhang, L.; Yu, K.; Eisenberg, A. Science 1996, 272, 1777-1779.

(4) Bhargava, P.; Zheng, J. X.; Li, P.; Quirk, R. P.; Harris, F. W.; Cheng, S. Z. D. Macromolecules 2006, 39, 4880-4888.

(5) Abbas, S.; Li, Z.; Hassan, H.; Lodge, T. P. Macromolecules 2007, 40, 4048-4052.

(6) Cui, H.; Chen, Z.; Zhong, S.; Wolley, K. L.; Pochan, D. J. Science 2007, 317, 647-650.

(7) Zamurovic, M.; Christodoulou, S.; Vazaios, A.; Iatrou, E.; Pitsikalis, M.; Hadjichristidis, N. Macromolecules 2007, 40, 5835-5849, and references therein.

(8) Hong, D.-J.; Lee, E.; Lee, M. Chem. Commun. 2007, 1801-1803.

(9) Jonas, A.-M.; Glinel, K.; Oren, R.; Nysten, B.; Huck, W. T. S. Macromolecules 2007, 40, 4403-4405.

(10) Lutz, J.-F.; Weichenhan, K.; Akdemir, Ö.; Hoth, A. Macromolecules 2007, 40, 2503-2508.

(11) Skrabania, K.; Kristen, J.; Laschewsky, A.; Akdemir, Ö.; Hoth, A.; Lutz, J.-F. Langmuir 2007, 23, 84-93.

(12) Hua F.; et al. Submitted for publication.

(13) Hua, F.; Jiang, X.; Zhao, B. Macromolecules 2006, 39, 3476-3479.

(14) Cheng G.; et al, Submitted for publication.

(15) Mao, G.; Sukumaran, S.; Beaucage, G.; Saboungi, M.-L.; Thiyagarajan, P. Macromolecules 2001, 34, 552-558.

(16) Yang, L.; Alexandrisdis, P. Langmuir 2000, 16, 4819-4829.

(17) Pedersen, J. S.; Gerstenberg, M. C. Colloids Surf. A: Physicochem. Eng. Asp. 2003, 213, 175-187.

(18) (a) Mortensen, K. J. Phys.: Condens. Matter 1996, 8, A103-A124. (b) Bang, J.; Viswanathan, K.; Lodge, T. P.; Park, M. J.; Char, K. J. Chem. Phys. 2004, 121, 11489-11500.

(19) Mortensen, K.; Pedersen, J. S. Macromolecules 1993, 26, 805-812.

(20) (a) Goldmints, I.; Yu, G.; Booth, C.; Smith, K. A.; Hatton, T. A. Langmuir 1997, 13, 3659-3664. (b) Chen, S.-H.; Liao, C.; Fratini, E.; Baglioni, P.; Mallamace, F. Colloids Surf. A: Physicochem. Eng. Asp. 2001, 183-185, 95-111. (c) Jain, N. J.; Aswal, V. K.; Goyal, P. S.; Bahadur, P. J. Phys. Chem. B 1998, 102, 8452-8458. (d) Pedersen, J. S. J. Chem. Phys. 2001, 6, 2839-2846.

(21) Svaneborg, C.; Pedesen, J. S. J. Chem. Phys. 2000, 112, 9661-9670.

(22) Bartlett, P.; Ottewill, R. H. J. Chem. Phys. 1992, 96, 3306-3318. MA702580V 